\title{
Design and implementation of an intelligent medicine box capable of detecting user's medication taking state
}

\author{
Tian-Li Hu ${ }^{1, a}$, Hong-Zhi Qian" b \\ ${ }^{1}$ Jilin Agricultural University, Changchun 130118, Jilin, China \\ aarcticfox129@163.com, b474117051@qq.com
}

\begin{abstract}
: in this paper, the main control part of the multifunctional intelligent medicine box is composed of AT89S52 microcontroller, ISD1700 voice chip, DS1302 clock chip and so on. The human-computer interaction is achieved by the LCD12864 and independent keyboard, and using the piezoelectric thin film sensors to detect the amount of medications, in order to add reminders. Connecting the mobile phone App with the medicine box through the Wi-Fi module to achieve the monitoring of the elderly medication while APP also provides users with online medical consultation platform and online pharmacy platform functions, it can make the multi-functional medicine box more intelligent, comprehensive and personalized service for the elderly to take medicine.
\end{abstract}

Keywords: intelligent medicine box, mobile phone App, Wi-Fi, AT89S52 MCU.

\section{The research background of intelligent medicine box}

China's National Bureau of Statistics data show that in 2015 China 65 years of age and older population of more than 130 million, accounting for $10.1 \%$ of the total population, population aging is serious. Nearly $58.1 \%$ of the elderly had chronic diseases. Due to chronic disease treatment cycle is long, long-term use of medication treatment [1]. The elderly memory gradually decline, temporary forget to take medicine, eat the wrong medicine or even repeated medication, seriously affecting the health. According to the State Food and Medication Administration, a statistical data show that China's annual 2.5 million people because of misuse of medications and damage to health, of which 0.2 million people died, is the national traffic accident deaths twice. In order to effectively improve the problem of medication for the elderly, the market appeared to help the elderly to take medicine smart kit.

\section{Analysis of existing intelligent medicine box}

At present the domestic market has been a lot of forms of smart kit, according to their main selling points can be divided into the following three categories:

(1) To operate simply for design purposes. This type of smart kit is usually used to partition the various types of medications in the box to distinguish the different space, mainly in order to facilitate the user sub-packaged medications, easy to confuse the medication to distinguish, in order to avoid Eat the wrong medicine or repeated medication. The disadvantage is that there is no initiative to remind the function of taking medicine, users tend to forget to take medicine, so the security is not high. 
(2) For portable design purposes [2]. This type of smart kit mainly for the purpose of portable, small size, easy to travel to carry use. The disadvantage is that the function is not comprehensive; intelligent degree is not enough; save the amount of less often need dressing.

(3) To remind for design purposes. This type of smart kit with a regular alarm to remind the medication, SMS reminders to take medicine, design patterns diversified. But its shortcomings are more prominent: for the elderly for the process of setting up complex and error-prone; one-way service users do not have feedback, that is, simply remind the way to get the feedback has been medication, often ringing but no medication Of the situation; and this part of the function can be completely through other tools such as mechanical alarm clock, smart phones and other alternative to complete.

\section{The Design of intelligent medicine box}

Based on the above analysis of the advantages and disadvantages of the existing market, the main use of intelligent kit for the elderly, but its consumer groups are often the children of the elderly. The market needs a simple operation, with medication information feedback of the high security of the smart kit to protect the elderly correct medication. So through the use of app to set the kit to remind the child to pick up the time to receive whether the medication feedback specifically for the elderly to serve the smart box and its app practical and urgent market demand. The expected kit function and the corresponding problem-solving table are as follows:

Table 1 Comparison table

\begin{tabular}{cc}
\hline Kit function & Solve the problem \\
\hline Multi alarm clock timing function & To achieve multiple reminders \\
\hline Family recording tips & Personalized recording, increased intimacy \\
\hline Intelligent detection of medication & Alarm after reminder \\
\hline A key call for help & Living alone for self-help \\
\hline Temperature and humidity intelligent \\
control & Medications are stored for long periods of \\
time \\
\hline $\begin{array}{c}\text { Suggesting that the medication is } \\
\text { insufficient }\end{array}$ \\
LCD & Timely Tim medicine \\
\hline Wi-Fi module communication & Intuitive grasp of the kit of information \\
\hline GSM module to send text messages & The kit is connected to the App \\
\hline
\end{tabular}

\section{The Structure and Principle of Intelligent medicine box}

To remind the elderly to take medicine multi-functional smart kit is a combination of mobile phone App set up more alarm clock, personalized voice reminder, remote monitoring reminders, feedback medication information and other multi-functional smart kit. The kit is shown in Figure 1, was cylindrical, $16 \mathrm{~cm}$ in diameter, $20 \mathrm{~cm}$ high. The medicine box is divided into medicine storage unit, taking medicine unit. 


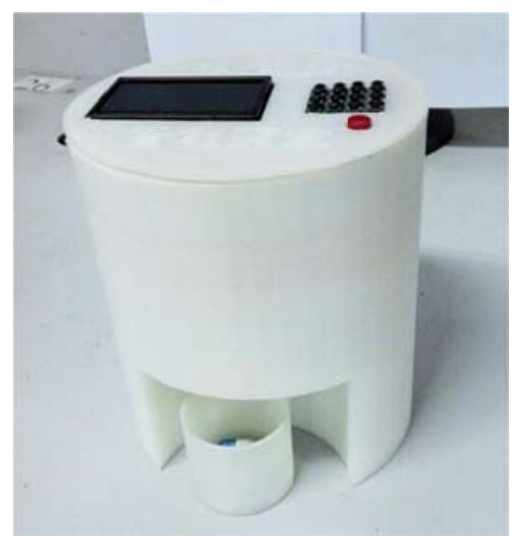

Fig.1 kit physical map

\subsection{Survival unit}

As shown in Figure 2, the physical diagram of the medication storage unit is composed of trays, center shafts and spaced trapezoidal grooves. The trapezoidal groove is connected to the center shaft and is detachable for easy cleaning and disinfection. Spaced equal trapezoidal groove will be divided into 24 units, one of the trapezoidal groove corresponding to the tray gap, the remaining 23 for the storage of medications, one-time storage of medications a week, greatly increased the amount of medications. Trap gap is designed for the medicine box medicine, the medication through the tray gap bit down to the bottom of the Sheng bowl to facilitate the use of the elderly. As long as the early morning, middle and late medications in a timely manner into the trapezoidal slot, you can ensure that the elderly by the amount of time to take the right medications.

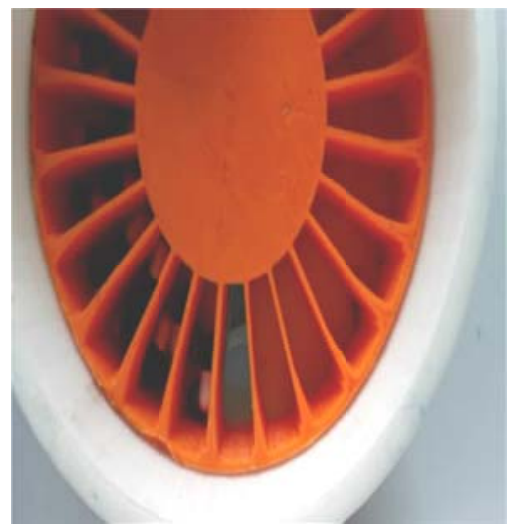

Fig.2 Survival unit physical map

Intelligent PCS module circuit schematic diagram shown in Figure 3. The system uses AT89S52 microcontroller as the master chip [3], high-performance 8-bit microcontroller, 32 two-way I / O port, burn the program, to achieve the control of each module. The control unit system as shown in Figure 4, the system power supply for the control unit to provide power; independent button to provide a good human-computer interaction environment and emergency situations can be a key call for help alarm function, easy to live alone in dangerous circumstances self-rescue. The use of pyro electric infrared sensor to detect whether the elderly on time to take medicine, when the first medication is not removed, the next medication will not be sent out; temperature and humidity sensor to detect the environment in the smart medicine box in order to control the heating (Or fan) work to effectively save the medication; Piezoelectric film sensor to detect the dose of the medication unit to allow the microcontroller to pay less than the amount of reminder [4]; use DS1302 clock chip to set the current time and multi-alarm clock timing function; ISD1700 Voice module to provide personalized recording function, to achieve user self-recording to alert the alarm, 
increase the intimacy; use Wi-Fi module to smart box and App communication [5]; use LCD12864 to display the current time, set the alarm interface, personalized tips Select and set the contact, as shown in Figure 5. Alarm circuit to provide low-dose voice prompts, GSM module to send text messages to set the contact.

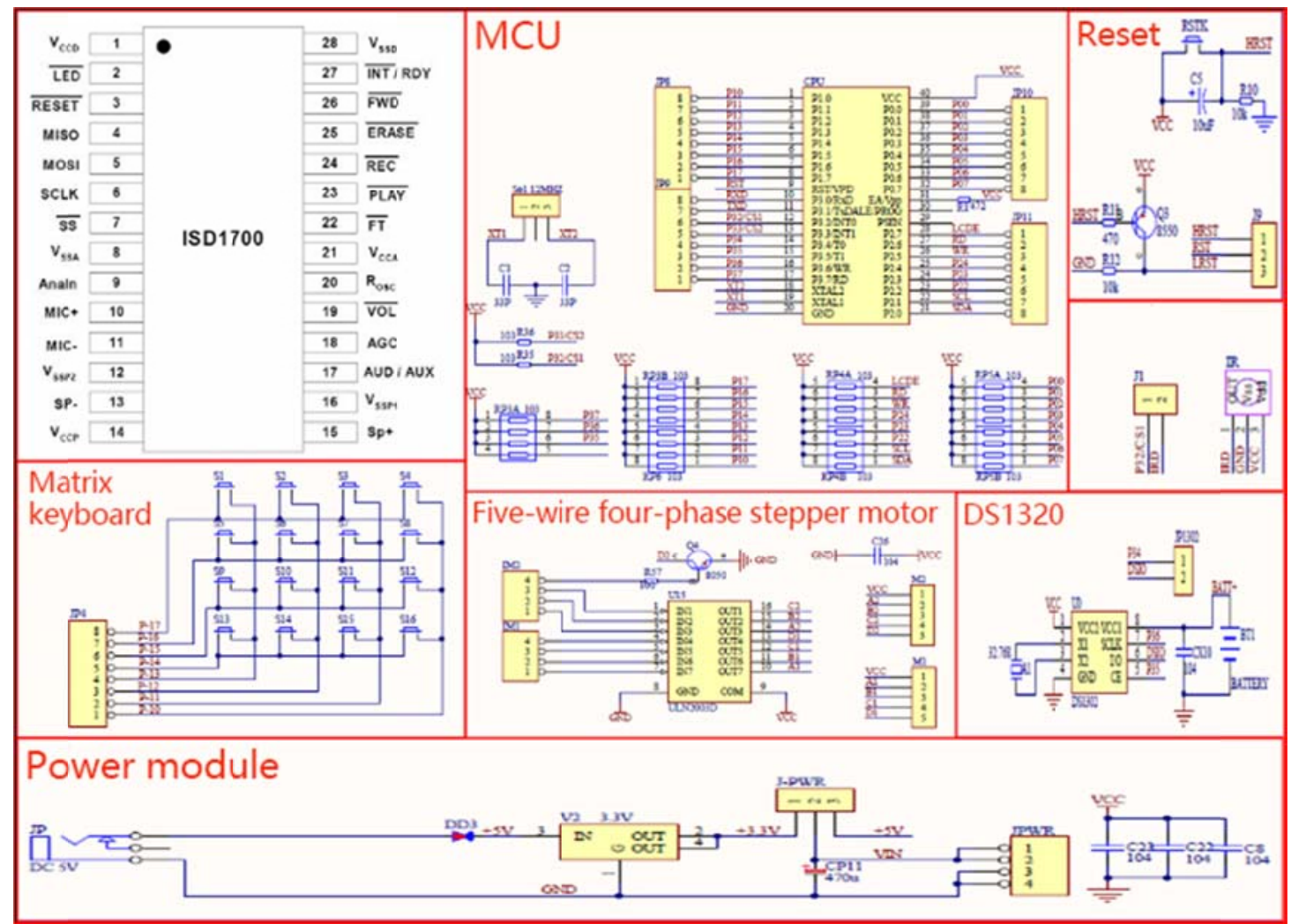

Fig.3 Circuit schematic

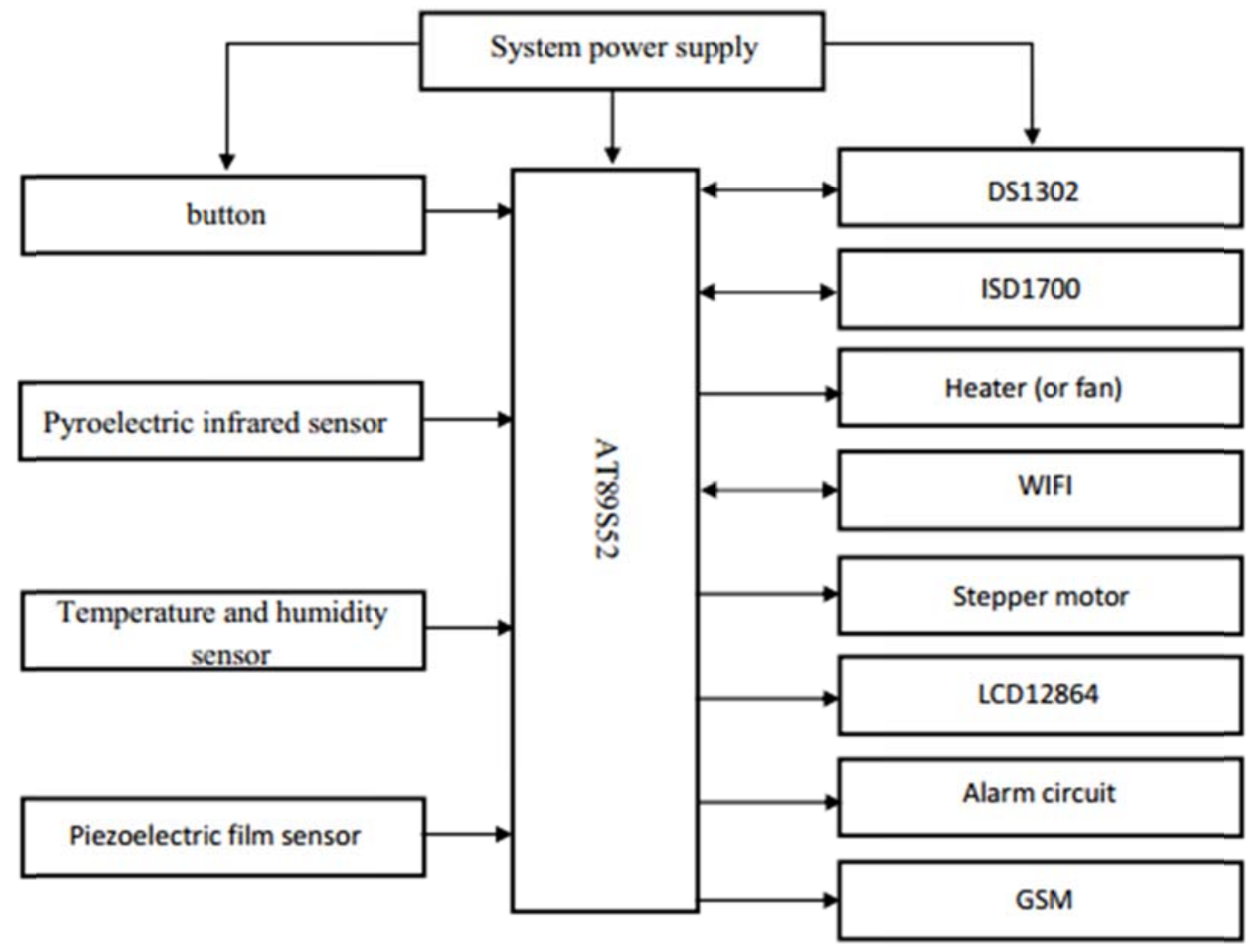

Fig.4 A general block diagram of the control unit system 


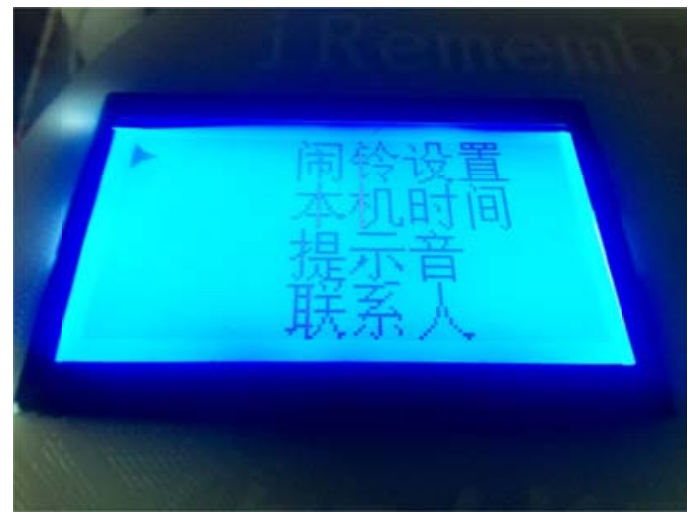

Fig.5 LCD12864Liquid crystal display

\subsection{Outlet unit}

The medication withdrawal unit consists of a bowl of pots and a pyroelectric infrared sensor. When the medication falls into the bowl, if the pyrolysis infrared sensor detects that the medication bowl is removed, the monolithic unit controls the ringing and stops Will send a non-medication message to the contact [6], on the contrary, will continue to ring for 15 minutes (time can be set), through the kit within the GSM module to send text messages to the contact [7]. The system through the Wi-Fi module to communicate with the mobile App, recorded in the APP on the taking of the medication. Multifunctional smart kit specific operation of the process shown in Figure 6.

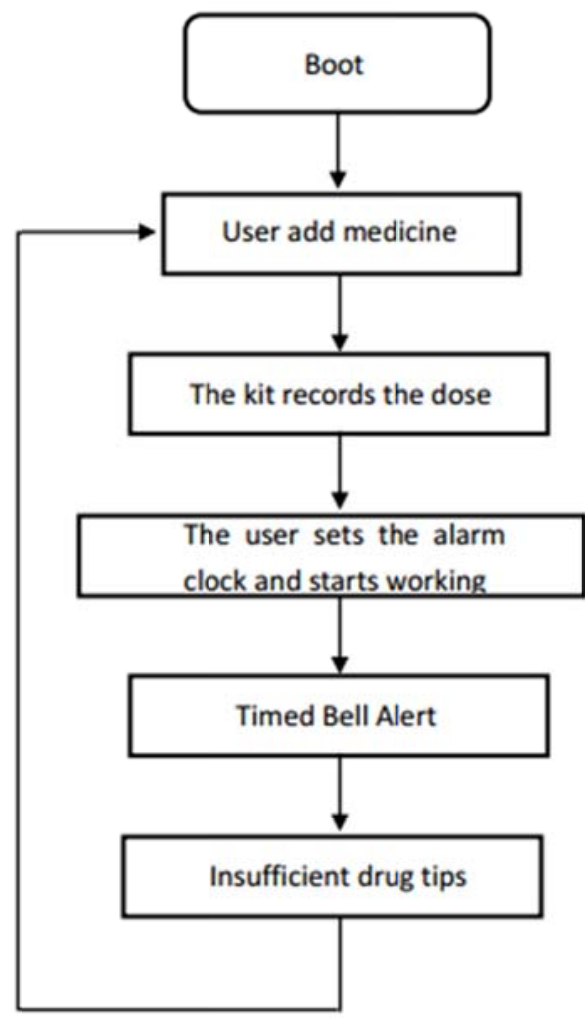

Fig.6 Operation process diagram

\section{The design of App function interface}

To remind the elderly to take medicine multi-functional smart kit will be mobile phone App and kit, through the Wi-Fi mobile phone and kit to connect [8], traditional kit App can only provide services for users with the kit to carry out the medication Box personalization and remote monitoring. And remind the elderly to take medicine multi-functional smart kit App interface features not only include the basic function of the home kit, medication records, news center of these traditional 
intelligent kit remote control function, but also for the current online purchase can't achieve the situation, App opened an online pharmacy platform for users and their nearby physical pharmacies to build online communication platform. At the same time App platform has medical information and doctors and patients interactive online platform, so that users can also consult online related to medical problems, so App can also be no smart kit for the user service. In order to more comprehensive protection of the elderly safe medicine, but also help the elderly more convenient to buy medications and daily health advice and maintenance. App interface function layout shown in Figure 7.
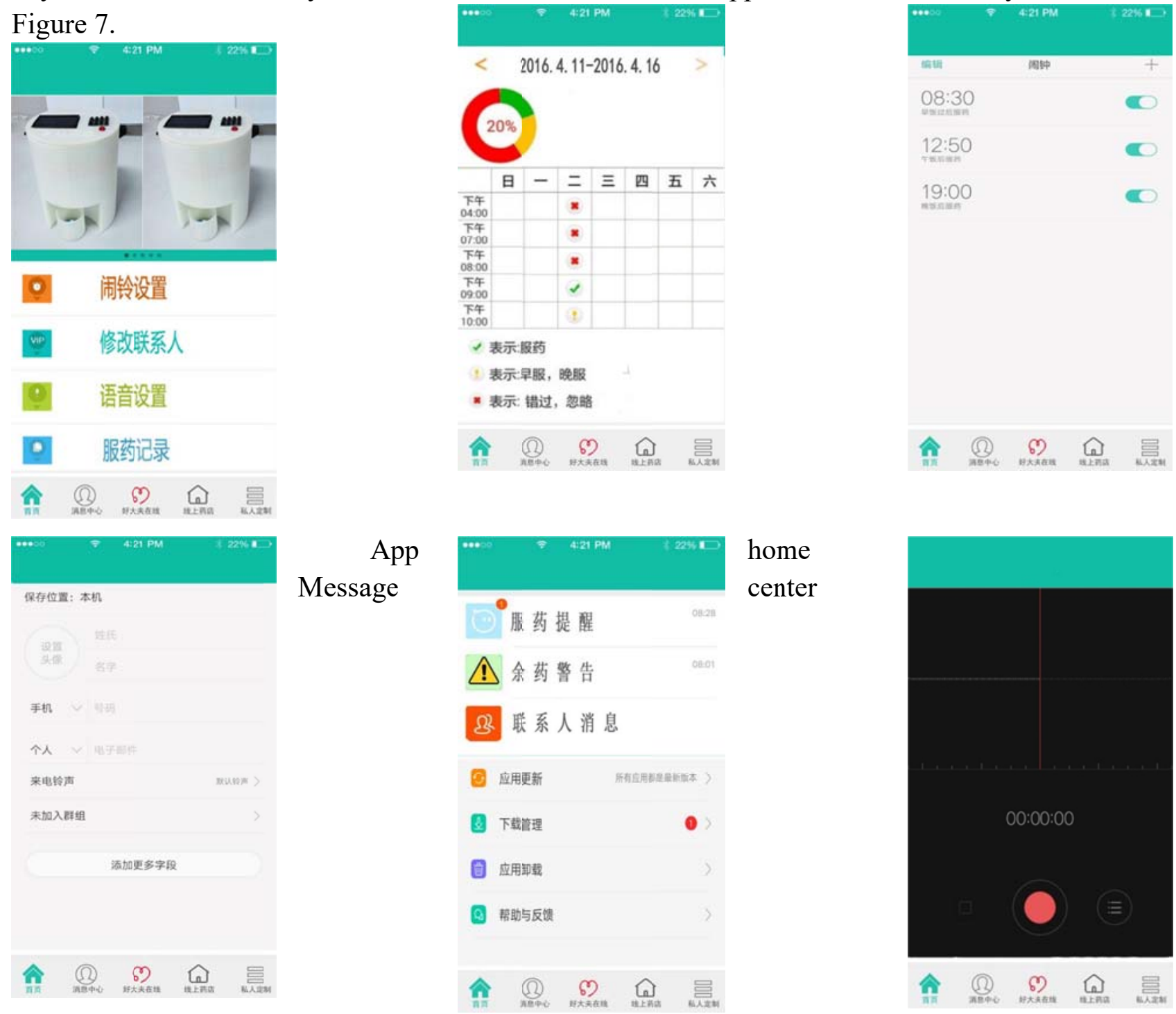

home center

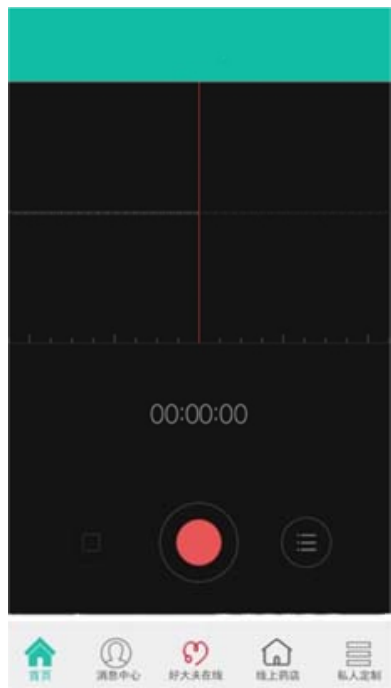

Personalized voice recording Medication record

Contact settings

Medication alarm settings

Fig.7 App interface function layout

\section{Summary}

Through a kind of to remind the elderly to take medicine multi-functional smart kit structure analysis and App function interface, a simple understanding of the work of multi-functional smart kit and with the use of App. It is mainly reminded to take medicine on time, the amount of medication and the medication temperature and humidity environment is guaranteed, personalized recording tips, less than reminder, emergency call for help and other functions. Even App intelligent connection kit to achieve the mobile terminal on the smart kit for remote settings, set the medication 
to remind the time, intelligent generation of medication records weekly, the user is not on time to take medicine and medication stock is insufficient by App intelligent reminder, online doctor consultation Platform and online pharmacy platform and other functions, more intelligent, comprehensive service for the elderly to take medicine.

\section{Acknowledgments}

This work by the 2016 Jilin Agricultural University National (provincial) College Students innovation and entrepreneurship program funded projects: Ai Rui Electronic Technology Co., Ltd. (386). Jilin Agricultural University doctoral research start funds (201621). Research and Application of Greenhouse Big shed Monitoring System Based on Internet of Things in Jilin Province (2015.184). By the Jilin Provincial Agricultural Science and Technology Network Collaborative Innovation Center (20160623016TC) to provide test sites and equipment.

\section{References}

[1] XIA Jinjun, YANG Liu, WU Zhiyuan, Optimized Design of Intelligent Kit for Elderly for User Experience [J], Packaging engineering, 2016.09.20.

[2] WANG Yuting, SHENG Jianping, A layered drop the form of medication design [J], Machinery Manufacturing, 2016.03.20.

[3] WU Yadong, SUN Ya, ZENG Guang, Based on AT89S52 intelligent voice to remind the kit design [J], Journal of Anyang Institute of Technology, 2014.03.20.

[4] LIU Kewang, GU Yongquan, WU Zongxiang, Piezoelectric film sensor production and calibration [J], Fluid Machinery, 1995.01.

[5] ZHANG Qiubo, Android phone and Wi-Fi based on the network timer switch design and implementation [D], Jilin University, 2015.06.01.

[6] JIA Qiang, Based on pyro electric infrared sensor circuit design [J], Electronic Technology and Software Engineering, 2015.02.05.

[7] QI Hao, WANG Li, WU Yi, et al. Based on the single-chip GSM SMS transceiver module design [J], Electronic Testing, 2012.03.05.

[8] Li Xiaoli, Smart kit [J], Packaging Engineering, 2015.01.20. 